\title{
Mapeamento sistemático: sistemas audiovisuais para o ensino de crianças com o transtorno do espectro autista
}

\author{
Systematic mapping: audiovisual systems for teaching children with \\ autism spectrum disorder
}

Mapeo sistemático: sistemas audiovisuales para la enseñanza de niños
con el trastorno del espectro autista

Rafael Moura Toscano $1, a$

rafaelmtoscano@gmail.com | http://orcid.org/o000-0003-1732-7600

Valdecir Becker ${ }^{2, b}$

valdecir@ci.ufpb.br | http://orcid.org/o00o-0002-0639-3875

\footnotetext{
${ }^{1}$ Universidade Federal da Paraíba, Pós-Graduação em Computação Comunicação e Artes. João Pessoa, PB, Brasil.

${ }^{2}$ Universidade Federal da Paraíba, Centro de Informática. João Pessoa, PB, Brasil.

a Graduação em Comunicação em Mídias Digitais pela Universidade Federal da Paraíba.

b Doutorado em Engenharia Elétrica pela Escola Politécnica da Universidade de São Paulo.
}

\section{Resumo}

Este artigo apresenta um mapeamento sistemático do estado de conhecimento das técnicas e dos métodos de utilização de sistemas audiovisuais, tais como vídeos, apps e jogos no processo de ensino de habilidades aos portadores do transtorno do espectro autista. O mapeamento contempla artigos de quatro bases de dados e classifica os resultados por meio dos problemas e soluções de pesquisa dos estudos. Como resultado, percebe-se o uso de soluções com foco em conteúdo, tecnologia e contexto para atingir problemas de habilidades sociais, mentalização, aspectos recreativos e acadêmicos. As soluções identificadas atuam em quatro cenários de interação e, por meio de medidas clínicas, acompanham a intervenção e mapeiam novas estratégias. Por fim, percebe-se a necessidade de integrar os processos de intervenção aos cenários de interação, além das particularidades de uso dos indivíduos com o intuito de construir sistemas audiovisuais modulares e adaptativos para o treinamento das habilidades sociais.

Palavras-chave: Sistemas audiovisuais; Habilidades sociais; Autismo; Crianças; Vídeos; Jogos. 


\begin{abstract}
This article presents a systematic mapping of the state of knowledge of techniques and methods of using audio and video systems, such as videos, apps and games in the process of teaching skills to people with autism spectrum disorder. The mapping comprises articles from four databases and classifies the results through the problems and research solutions of the studies. The results reveal the use of solutions focused on content, technology and context to reach problems of social skills, mentalization, recreational and academic aspects. The identified solutions act on four interaction scenarios and by means of clinical measures they accompany the intervention and map out new strategies. Finally, we observe there is a need for integrate the intervention processes into the interaction scenarios, as well as the use of the particularities of individuals should be take into account in order to build modular and adaptive audiovisual systems for training social skills.
\end{abstract}

Keywords: Audiovisual systems; Social skills; Autism; Children; Videos; Games.

\title{
Resumen
}

Este artículo presenta un mapeo sistemático del estado de conocimiento de las técnicas y de los métodos de utilización de sistemas de audio y vídeo, tales como videos, aplicaciones y juegos en el proceso de enseñanza de habilidades a los portadores del trastorno del espectro autista. El mapeo comprende artículos de cuatro bases de datos y clasifica los resultados por medio de los problemas y soluciones de investigación de los estudios. Como resultado, se percibe el uso de soluciones con foco en contenido, tecnología y contexto para alcanzar problemas de habilidades sociales, mentalización, aspectos recreativos y académicos. Las soluciones identificadas actúan en cuatro escenarios de interacción y por medio de medidas clínicas acompañan la intervención y mapean nuevas estrategias. Por último, se percibe la necesidad de integrar los procesos de intervención a los escenarios de interacción, además de las particularidades de uso de los individuos con el propósito de construir sistemas audiovisuales modulares y adaptativos para el entrenamiento de las habilidades sociales.

Palabras clave: Sistemas audiovisuales; Habilidades sociales; Autismo; Niños; Vídeos; Juegos.

Contribuição dos autores:

Concepção e desenho do estudo: Rafael Moura Toscano, Valdecir Becker.

Aquisição, análise ou interpretação dos dados: Rafael Moura Toscano, Valdecir Becker.

Redação do manuscrito: Rafael Moura Toscano.

Revisão crítica do conteúdo intelectual: Valdecir Becker.

Declaração de conflito de interesses: não há.

Fontes de financiamento: não houve.

Considerações éticas: não há.

Agradecimentos/Contribuições adicionais: não há.

Histórico do artigo: submetido: 26 jan. 2018 | aceito: 11 mar. 2019 | publicado: 28 jun. 2019.

Apresentação anterior: não houve.

Licença CC BY-NC atribuição não comercial. Com essa licença é permitido acessar, baixar (download), copiar, imprimir, compartilhar, reutilizar e distribuir os artigos, desde que para uso não comercial e com a citação da fonte, conferindo os devidos créditos de autoria e menção à Reciis. Nesses casos, nenhuma permissão é necessária por parte dos autores ou dos editores. 


\section{Introdução}

As tecnologias da informação e comunicação (TIC) apresentam uma crescente representatividade em contextos específicos, como nos de processos educacionais e de intervenções clínicas, especialmente em áreas relacionadas às necessidades motoras, cognitivas e psicossociais dos indivíduos. Dentro desse escopo podemos destacar a utilização de tecnologias de áudio e vídeo digital, tais como vídeos, aplicativos (apps) e jogos, para o ensino de competências e habilidades a crianças com transtorno do espectro autista (TEA). A questão desta pesquisa surge exatamente dos déficits sociais, comportamentais e de comunicação inerentes a estes indivíduos.

Diante desse cenário, surgem questões relacionadas: (a) à forma como acontece o processo de aprendizado de habilidades, competências e comportamentos sociais em portadores de TEA; (b) aos efeitos desse processo ao ser mediado por mídias digitais e interativas; (c) a como medir e quantificar o aprendizado; e (d) à identificação de quais os principais formatos e dispositivos de interação nele utilizados. Ou seja, esta pesquisa se propõe a verificar o estado do conhecimento dos processos de intervenção para o desenvolvimento cognitivo e comportamental de habilidades a portadores de TEA, pelo recorte das tecnologias assistivas de áudio e vídeo.

O processo de digitalização das mídias fomenta novas possibilidades de criação e distribuição de informação. Precisamente, as tecnologias de áudio e vídeo tornam possível expandir as lógicas de exposição linear a estruturas de interação cada vez mais complexas. Nesse cenário, a classificação sobre o que é vídeo, jogo e software torna-se um processo exaustivo, nem sempre com retornos significativos. Sendo assim, este trabalho utiliza a terminologia de sistemas audiovisuais para definir qualquer solução de mídia que integre aspectos técnicos de vídeos, jogos, rádio, televisão, sensores e softwares ${ }^{1}$. A complexidade dos sistemas pode variar desde a simples reprodução de mídia até interações mediadas por dispositivos hápticos, sensíveis ao toque, ou interfaces cerebrais.

Como objetivo geral, o presente artigo apresenta um mapeamento sistemático das soluções desenvolvidas no período de 2015 a 2017 para uso da tecnologia assistiva e audiovisual no ensino de competências e habilidades funcionais. O perfil do mapeamento é interdisciplinar, compreendendo ciências humanas, sociais, biológicas e exatas. Como objetivos específicos, visa-se: I) Elencar produções científicas dos últimos dois anos em quatro bases de dados relevantes à computação, à multimídia e à interdisciplinaridade; II) Identificar e relacionar problemas, soluções de pesquisa e estratégias de medição do processo de aprendizado recorrentes nos artigos; III) Avaliar o uso de modelos de execução de atividade como base de ensino-aprendizado; IV) Relacionar as soluções encontradas e apontar usos potencias.

Este trabalho está dividido do seguinte modo: em uma seção são apresentados conceitos gerais sobre autismo, aprendizado e sistemas audiovisuais; na seção seguinte, os métodos de pesquisa do mapeamento são detalhados; outra seção é composta pelos resultados da pesquisa categorizados de acordo com duas perspectivas: problemas e soluções; a seção posterior apresenta as análises e discussões sobre os resultados. Finalmente, a última seção apresenta as conclusões e alguns apontamentos para trabalhos futuros.

\section{Fundamentação teórica}

Nesta seção, apresentam-se os conceitos fundamentais em que se baseia a realização desse mapeamento.

\section{Autismo}

O transtorno do espectro autista (TEA) pode ser compreendido como um distúrbio neurobiológico do cérebro, caracterizado pelo comprometimento da interação social, comunicação e presença de padrões repetitivos e estereotipados ${ }^{2}$. Esta compreensão atual é fruto de um longo percurso desenvolvido pela comunidade acadêmica. A primeira discussão sobre o distúrbio autístico surge na década de 1940, por 
meio dos estudos de Kanner, que, a partir de 11 casos clínicos, conceituou uma síndrome cujos principais sintomas são: inabilidade social, problemas de linguagem, de comunicação e necessidade de padrões repetitivos ${ }^{2}$. No mesmo período, outro pesquisador, Hans Asperger, também conceituou indivíduos com características atípicas no comportamento².

A compreensão sobre a natureza das características dos portadores do autismo passou por diversas hipóteses. Mintz ${ }^{2}$ realiza um levantamento histórico dessa evolução e destaca que, inicialmente, o autismo foi considerado um transtorno emocional ou comportamental, em seguida, uma alteração cognitiva e, mais recentemente, a síndrome se popularizou como um distúrbio do neurodesenvolvimento.

As pesquisas ${ }^{2,3}$ destacam que, a partir dos anos 1980, por meio do terceiro Manual de Diagnóstico e Estatístico de Transtornos Mentais (DSM-III), o autismo ganhou uma conotação separada dos retardos mentais, porém continuou limitado à compreensão dos fatores neurológicos do distúrbio. Com as versões seguintes do manual, o conceito sofreu alterações, de modo que o autismo passou por subcategorizações até culminar na abordagem da quinta versão. $\mathrm{O}$ manual ${ }^{4}$ compreende o autismo como um espectro que engloba o transtorno de Asperger, o transtorno desintegrativo da infância, o transtorno de Rett e o transtorno global do desenvolvimento. Além disso, é caracterizado por déficits na comunicação e interação social, padrões repetitivos e restritos de comportamento, interesses e atividades.

Essa versão elimina a nomenclatura adotada anteriormente e admite a existência de variação de atributos que constituem a noção de espectro do transtorno. Além da abordagem na perspectiva do neurodesenvolvimento, a pesquisa de Mintz $^{2}$ destaca a importância de estudos atuais que reconhecem o transtorno autista como um distúrbio neurobiológico. Para $\mathrm{Mintz}^{2}$, apenas com uma abordagem interdisciplinar e transdisciplinar é possível compreender os subfenótipos biológicos e neurológicos dos portadores do transtorno e, desse modo, desencadear soluções de intervenção que promovam qualidade de vida para o indivíduo e seus responsáveis.

Visto que o transtorno do espectro autista é compreendido como um distúrbio do neurodesenvolvimento, é importante entender como se dá o processo de construção de conhecimento e desenvolvimento cognitivo.

\section{Aprendizado: a relação corpo e mente}

Vygotsky ${ }^{5}$ considera a aprendizagem como um processo cognitivo que teria lugar dentro e fora da mente das pessoas. Para o autor, essa internalização do saber ocorre por meio da relação com o meio, com outros indivíduos mais experientes e, principalmente, pelo acervo de competências e habilidades cognitivas que o indivíduo já dispõe como ambiência para todo esse curso.

O processo de aprendizagem é fruto de uma ação intencional promovida pela relação social e contextual para desenvolver processos de captação, integração, elaboração e expressão da informação ${ }^{6}$. Para o autor, essa educação cognitiva é baseada na capacidade de desenvolver competências de resolução de problemas por meio de processos e subprocessos cognitivos que são trabalhados de modo estruturado e cumulativo. Desta forma, as habilidades cognitivas estão relacionadas diretamente com a maneira como acontece a interação com os outros num ambiente específico de solução de problemas ${ }^{5,6}$.

Integrando também a essa discussão os estudos de Leontyev ${ }^{7}$ e de Sirlene ${ }^{8}$, podemos sintetizar que a construção do aprendizado é centrada em três tópicos gerais: a) na necessidade ou no motivo7; b) na zona proximal, isto é, a relação entre a capacidade real e potencial de um indivíduo ${ }^{5}$; c) nos interesses do indivíduo ${ }^{8}$. Sendo assim, é possível resumir a aprendizagem como o contato com a modelagem de formas e significados que buscam trazer ao indivíduo motivos e finalidades pelos quais é importante absorver determinados conteúdos, ou melhor, fomentar a percepção de valor sobre internalizar o conhecimento.

A relação corpo, mente e contexto pode ser encontrada também no estudo ${ }^{9}$, uma vez que os autores compreendem a transmissão, ou hereditariedade, de conhecimento como processos de aprendizado social 
e imitação de habilidades. Neste sentido, a compreensão do desenvolvimento de habilidades sociais se torna relevante para que o indivíduo com o transtorno do espectro autista (TEA) viva na plenitude social e cultural de seu contexto.

Por fim, a pesquisa ${ }^{10}$ destaca a importância da relação corpo e mente e acrescenta a perspectiva de que no objeto técnico existe um estado potencial do ser humano, isto é, uma expansão da corporificação e das habilidades do indivíduo. Segundo os autores, a criança com problemas cognitivos vivencia nos artefatos digitais uma espécie de autorregulação, e por abdução, entende que também podem se autorregular.

\section{Modelos de execução de atividade}

O aprendizado comportamental a partir de modelos comportamentais é tema recorrente na psicologia, na pedagogia e na sociologia. $\mathrm{O}$ estudo ${ }^{11}$ realiza um levantamento histórico dos trabalhos feitos pela psicologia e motricidade que relacionam o déficit da capacidade de imitação ao autismo. Segundo os autores, pesquisas com essa temática tiveram início na década de 1970 e discutiram, até o final da década de 1990, posicionamentos distintos acerca das implicações de relação entre autismo e imitação.

Apesar das diferentes visões sobre a intensidade de causa e efeito, a partir da década de 1990, estudos científicos têm apontado para uma certa unanimidade sobre a questão de que portadores de TEA apresentam um déficit na capacidade motora de imitação nos primeiros anos. Além disso, acredita-se também que uma intervenção direcionada seria capaz de desenvolver aspectos importantes, como a intersubjetividade, que fomenta uma série de competências e habilidades para o convívio social do portador.

O levantamento ${ }^{11}$ nos ajuda a compreender os modelos de atividade como abordagem eficaz ao desenvolvimento pessoal dos portadores do autismo. Inclusive, em alguns casos, a intervenção direcionada ao desenvolvimento da capacidade de imitar, e ser imitado, gera uma via de comunicação e até mesmo de afetividade. Para os autores do estudo ${ }^{11}$, o movimento age como o mensageiro da emoção, isto é, o trabalho com a imitação processual motora desencadeia, além de impactos externos e comportamentais, a capacidade cognitiva de perspectiva sobre os outros indivíduos que o cercam.

A geração de modelos não é restrita à relação interpessoal direta entre indivíduos. Ao contrário, a mediação desse processo ganha novas possibilidades com a incorporação das TIC. Desde o simples registro de momentos em representações estáticas em imagens, ao fluxo dinâmico de eventos em vídeos, e contemplando softwares interativos que integram sensores à experiência de uso, a utilização de TIC tem se tornado relevante no processo de ensino de habilidades a portadores de TEA.

\section{Métodos}

O método de investigação desta pesquisa é o mapeamento sistemático (MS) da literatura. Um MS, de modo geral, é composto pelas etapas de planejamento, formulação das questões de pesquisa, definição dos critérios de inclusão e exclusão, busca e revisão dos estudos. O MS visa estruturar uma revisão na literatura com o objetivo de verificar e comparar soluções ou experimentos científicos.

Avaliando o cenário de mídia e aprendizado para crianças portadoras de TEA, não foram identificadas revisões sobre quais os formatos de interação utilizados e qual o seu contexto de utilização. Sendo assim, este MS tem o objetivo de atender a essa lacuna. A pesquisa foi conduzida de acordo com as diretrizes propostas por Kitchenham e Charters ${ }^{12}$ e seguiu a abordagem de classificação (problemasolução) baseada em na pesquisa ${ }^{13}$. 


\section{Questões de pesquisa}

As questões deste estudo foram desenvolvidas de acordo com os critérios do protocolo de pesquisa Pico (Population, Intervention, Comparison, Outcomes). Essa abordagem, comum às ciências médicas, é apontada pelo estudo ${ }^{12}$ como aplicável às áreas da computação, multimídia e estudos interdisciplinares, dado que fornece uma visão estruturada e sistêmica da pesquisa baseada em evidências. A partir das quatro dimensões que compõe o Pico foi desenvolvido o protocolo desta pesquisa. (Ver quadro 1)

\section{Quadro 1-Protocolo Pico}

\begin{tabular}{|l|l|}
\hline Population & $\begin{array}{l}\text { Estudos atuais sobre o aprendizado e desenvolvimento de } \\
\text { habilidades e comportamentos sociais em crianças autistas. }\end{array}$ \\
\hline Intervention & Uso de vídeos e jogos como estratégia de ensino. \\
\hline Comparison & Uso de modelos de atividade. \\
\hline Outcomes & Principais usos e aplicações destas mídias e seus impactos. \\
\hline
\end{tabular}

Fonte: Os autores (2019).

Conforme sintetizado no Quadro 1, Population neste estudo consiste no mapeamento de pesquisas primárias referentes ao ensino de competências e habilidades para crianças com o TEA. O item Intervention representa o recorte temático sobre o interesse direcionado às soluções que utilizem vídeos ou jogos digitais, reconhecidas neste trabalho como sistemas audiovisuais. Já Comparison refere-se à avaliação desta pesquisa sobre o uso ou não de modelos de atividade de acordo com a literatura. Por fim, o item Outcomes representa o resultado pretendido por esta pesquisa, isto é, mapear qualquer uso de vídeos e jogos no ensino de habilidades para os portadores de TEA e quais os impactos desse uso. Com base nos tópicos apresentados, foram formuladas as seguintes questões de pesquisa:

QP1: Como vídeos e jogos são utilizados no ensino de habilidades e competências para portadores de TEA, e quais os impactos dessa abordagem?

QP2: Como medir e quantificar o processo de aprendizado?

\section{Processo de busca}

Os termos e sinônimos considerados na construção da string de busca foram obtidos por meio da relação dos principais termos nas questões de pesquisa (Pico) e palavras-chave em um grupo de artigos selecionados previamente ${ }^{2,14-17}$. Os itens escolhidos foram: Autism, video, skill, game e exergaming e foram dispostos dentro das quatro bases em uma sentença 'autism AND video AND skill AND (game OR exergaming)'.

A construção dessa sentença comporta itens gerais como: a) Autism, termo reduzido para nomear o transtorno. A variação composta ASD - Autism Spectrum Disorder ou em português TEA - Transtorno do espectro autista já está acobertada pelo termo isolado; b) Video, termo abrangente que engloba vídeo digital, câmeras de vídeo, Video modelling, videogame, entre outros; c) Skill, o termo utilizado para habilidades sociais, habilidades cognitivas, habilidades e competências; d) Game OR exergaming, esta variação foi utilizada porque alguns autores reconhecem mídias interativas que promovem o engajamento físico como jogos ativos e outros autores utilizam o termo exergame para atribuir o mesmo sentido. De modo a agrupar um número maior de estudos, foram utilizadas as duas terminologias. As fontes de busca deste trabalho consistiram nas seguintes bases de dados: Scopus, Springer, IEEE Explorer e ACM Library. Estas bases foram escolhidas por serem relevantes no campo da computação, multimídia e interdisciplinaridade. Além da string de busca foram adicionados os filtros: (a) artigos em inglês (b) publicados em periódicos (c) no período de 2015 a 2017. 


\section{Critérios de inclusão e exclusão}

Para realizar a seleção dos estudos, foram definidos critérios de inclusão e exclusão. Assim, os estudos foram incluídos no MS por cumprir os seguintes critérios de inclusão (I):

I-1: Artigos referentes a estudos primários;

I-2: Versão completa do estudo disponível;

I-3: O estudo deve tratar objetivamente da utilização de sistemas audiovisuais no ensino de habilidades a crianças portadoras do TEA.

Sobre o item I-3, não foi delimitada uma faixa-etária ou conceito referente ao termo criança. Esta pesquisa reconheceu a nomenclatura adotada por cada autor. Sendo excluídos apenas trabalhos que abordaram um público de adolescentes, adultos ou idosos.

Em relação aos critérios de exclusão (E), um estudo foi excluído se:

E-1: Negar qualquer critério de inclusão, sendo obrigatório o registro do critério negado;

E-2: Estiver duplicado ou segmentado, relatando de forma parcial a mesma pesquisa. Neste caso, apenas a publicação mais recente foi considerada.

\section{Seleção e classificação dos estudos}

Como primeiro resultado, foram identificados 349 artigos, dispostos da seguinte maneira: 19 registros recuperados da Scopus, 304 da Springer, 23 da IEEE Explorer e dois da ACM Library. O processamento dos estudos ocorreu em duas partes: a aplicação dos critérios de busca e a classificação por categorias temáticas.

Na primeira etapa foram lidos os itens: título, palavras-chave, resumo e conclusão. Ao término da inspeção dos critérios de inclusão e exclusão foram considerados dezenove (19) estudos para o mapeamento (Tabela 1).

Tabela 1 - Aplicação dos critérios de busca

\begin{tabular}{lccccc}
\hline Bases & Resultados & \multicolumn{5}{c}{ Critérios } \\
\hline ACM & & Duplicado & Irrelevante & Indisponível & Aprovado \\
IEEE & 2 & 0 & 2 & 0 & 0 \\
Scopus & 24 & 0 & 23 & 0 & 1 \\
Springer & 19 & 0 & 10 & 1 & 9 \\
\hline Total & 304 & 5 & 294 & 0 & 9 \\
\hline
\end{tabular}

Fonte: Os autores (2019).

Os artigos finais foram classificados pelo contexto dos problemas de pesquisa (P) e das soluções propostas. Os problemas foram dispostos da seguinte maneira:

Métodos (P1): Discussões e comparações sobre práticas metodológicas.

Habilidades sociais: Intervenções no ensino de competências e comportamentos comuns na vida social. São elas: Gerais (P2); Mentalização (P3); Recreativa (P4); Acadêmica (P5);

Em seguida, os mesmos artigos foram revisados e classificados de acordo com as soluções (S) apresentadas.

Conteúdo: Estratégias em que o conteúdo ofertado pela solução midiática é o foco da solução. São elas: Modelos em vídeo (S1); Personalização do usuário (S2);

Tecnologia (S3): Estratégias em que o suporte de interação humano-computador é objeto central da solução.

Contexto (S4): Estratégias em que a solução é parte integrante de uma abordagem maior de intervenção. 


\section{Resultados}

Nesta seção são apresentados os resultados do mapeamento sistemático por meio das categorias de problemas e soluções.

\section{Problemas}

O primeiro filtro de extração dos dados se delimita a mapear as questões de pesquisas recorrentes nos trabalhos e agrupá-las em categorias comuns.

\section{Métodos}

A categoria de métodos comporta os artigos ${ }^{18-21}$ que apresentam estudos que concentram as discussões na avaliação de métodos e formatos para o processo de intervenção. As pesquisas relatam as diferenças entre o aprendizado em grupo e individual ${ }^{18}$; soluções digitais e híbridas por meio de experiências tangíveis ${ }^{20}$; competências necessárias ao uso de soluções midiáticas para intervenção ${ }^{18,20}$; potencialidade das mídias para 0 ensino ou extinção de comportamentos ${ }^{18,19}$; abordagem centrada em interesses em oposição a fins terapêuticos ${ }^{21}$.

Dentre os comparativos, podemos destacar as discussões acerca da modelagem em vídeo, ou melhor da estratégia de Video Modelling (VM). O estudo ${ }^{18}$ discute os impactos da variação da reprodução dos vídeos por meio da televisão em comparação aos dispositivos móveis. A pesquisa destaca que a capacidade de reprodução do modelo de atividade foi identificada primeiro nos participantes que utilizaram a TV. Para o estudo $^{18}$, o consumo em grupo, somado ao tamanho da tela, garante o impacto maior pela TV. Por outro lado, a capacidade de manutenção e generalização do comportamento foi mais eficiente pelo tablet (dispositivo móvel). Neste caso, a pesquisa aponta a assimilação duradoura pela maior facilidade de manipular o tempo (replay) por meio da interação nos dispositivos móveis.

Outro comparativo presente é a relação entre os modelos em vídeo e Social Stories (SS). O SS é uma estratégia em que fatos ou eventos são descritos por meio de frases e imagens de apoio. $\mathrm{O}$ autor da pesquisa ${ }^{19}$, destaca que o SS apresenta uma predisposição a intervir na redução de comportamentos enquanto a VM costuma fazê-lo na adição. As competências requeridas para o uso das mídias durante a intervenção também foram analisadas. A VM, por se tratar de um registro em vídeo de um ambiente real, foi aceito com mais facilidade. Já os SS apresentaram empecilhos nos testes porque exigiam dos participantes a capacidade de leitura e associação de imagens pictóricas.

Sobre a relação entre competências e consumo de mídia, a pesquisa ${ }^{20}$ retrata o uso de mídias híbridas, ou tangíveis, para o processo de intervenção em portadores que apresentam grande dificuldade de associação cognitiva e motora. Nesses casos, a pesquisa ${ }^{20}$ sugere que interfaces de associação física e virtual (digital) possibilitam o discurso de conversação, peça imaginativa, e habilidades de tomada de perspectiva a indivíduos com estágios mais rudimentares de desenvolvimento. Além destes tópicos, é possível identificar que, da mesma forma que o indivíduo deve aprender com a mídia, o sistema e os criadores de conteúdo devem aprender com o usuário (portador de TEA) ${ }^{21}$.

\section{Habilidades sociais}

A categoria de habilidades sociais é composta pelos trabalhos ${ }^{15,22-35}$. As pesquisas discutem as dificuldades cognitivas, emocionais, recreativas, acadêmicas, e como estas variáveis impactam a capacidade de interação social dos indivíduos com TEA. Segundo os estudos, as intervenções focadas nessas variáveis compõem um acervo de habilidades que possibilitam vida social funcional aos indivíduos. As pesquisas ${ }^{26,27,29}$ apontam o uso de vídeos e jogos como estratégia de intervenção como um todo. Deste modo, esses estudos foram classificados como gerais. Já as demais pesquisas ${ }^{15,22-25,28,30-35}$ apontam os parâmetros de mentalização, habilidades recreativas e acadêmicas como atributos que fomentam a interação social. 
Mentalização

Esta categoria comporta os seguintes estudos ${ }^{25,30,32-35}$. As habilidades de mentalização são retratadas como a capacidade de atribuir estados mentais, como crenças, pensamentos, sentimentos, planos e intenções, a si mesmo e a outros ${ }^{34}$. Essencialmente, essas pesquisas tentam promover a cognição e habilidades comportamentais que são consideradas essenciais para melhorar as habilidades sociais em longo prazo.

Entre os estudos, percebem-se discussões sobre: o estímulo e reconhecimento à produção de elogios ${ }^{25}$; reconhecimento de ações e emoções discretas ${ }^{30,32}$; controle e aprendizagem emocional ${ }^{33,35}$; atenção conjunta e processamento do rosto ${ }^{34}$. De modo geral, os estudos apontam os déficits de reconhecimento de expressões e estados emocionais dos portadores de TEA e destacam subcategorizações desta questão de interação social, de modo a intervir especificamente nas variáveis isoladas para alcançar o desenvolvimento global.

\section{Recreativa}

As habilidades recreativas são abordadas pelas pesquisas ${ }^{15,22,23,28,31}$ e consistem em capacidades como atenção, controle, equilíbrio, deslocamento de objetos e jogo simbólico. Os estudos desta categoria se dividem nos temas: atraso no desempenho global do jogo de simulação ${ }^{22}$; problemas motores e habilidades de movimento como: controle, equilíbrio e locomoção de objetos ${ }^{15}$; baixo interesse por atividades recreativas individuais ou em pares ${ }^{23,28,31}$.

\section{Acadêmica}

Entre as pesquisas levantadas apenas o artigo ${ }^{24}$ aborda a perspectiva de ensino-aprendizagem de conhecimentos acadêmicos. Nesse artigo, são abordadas as dificuldades de construção do conhecimento racional, segmentado e cumulativo do ensino formal; além disso, ele traz apontamentos sobre como intervenções mediadas por dispositivos digitais podem criar interesse nas temáticas escolares.

\section{Soluções}

O segundo filtro de extração dos dados absorve as hipóteses e soluções de pesquisas recorrentes nos trabalhos analisados e agrupa os resultados em categorias comuns.

\section{Conteúdo}

Os trabalhos que compõem esta categoria são ${ }^{18,19,23-25,28-33,35}$. A perspectiva de conteúdo pode ser subdividida em duas abordagens centrais: A utilização de Video Modelling (VM) ${ }^{18,19,23,25,28-31}$; e a personalização do conteúdo por meio de interesses e requisitos dos portadores $24,32,33,35$.

As soluções de VM são utilizadas para desencadear o aprendizado observacional e direcionar comportamentos verbais e não verbais como: estímulo à promoção e ao reconhecimento de elogios ${ }^{25}$; atividades de recreação individual ou em pares $^{18,19,23,28,31}$; sequência de comportamentos motores encadeados $^{28}$; reconhecimento de expressões e estados mentais ${ }^{30}$.

Já a personalização de conteúdo foi utilizada para: o ensino de temáticas escolares²4; o uso de Serious Games que permitem responsáveis e terapeutas adicionarem camadas e filtros graduais de personalização para o usuário ${ }^{33,35}$; a formulação de sistema com acervo e contexto dinâmico de representação de fala e movimentação para o ensino de estados emocionais ${ }^{33}$. 


\section{Tecnologia}

Os trabalhos que compõem esta categoria são $0^{15,20,22,34}$. As soluções desse grupo propõem interfaces de interação entre o homem e o computador específicas para promover a intervenção no ensino das habilidades aos portadores de TEA. Entre as interfaces estão: o uso de realidade aumentada para interação com objetos físicos ${ }^{22}$; câmeras e sensores de movimento corporal para o uso em jogos ativos, isto é, jogos que promovem atividades corpóreas no instante de uso da mídia ${ }^{15}$; sensores de rastreamento do olhar em monitores, para identificar e promover estímulos ao reconhecimento de expressões faciais ${ }^{34}$; interfaces tangíveis que permitem ao usuário a manipulação de objetos físicos para manipular o conteúdo abarcado no software ${ }^{20}$.

\section{Contexto}

As pesquisas desta categoria ${ }^{21,26,27}$ apresentam soluções que compreendem a mídia como parte integrante de um pacote de intervenções, isto é, parte de um programa de intervenção terapêutica maior. Apesar das pesquisas apresentadas em outras seções se relacionarem também com abordagens paralelas, os trabalhos focados em contexto apresentam um escopo maior do que o impacto direto pelo uso da mídia.

Estas pesquisas incorporam questões como: inclusão dos portadores de TEA no processo de criação dos conteúdos $^{21}$; atuação da atividade mediada por familiar como estratégia para promover melhorias no convívio social, isto é, utilizar o potencial lúdico da mídia para fornecer ao portador e seu familiar um ambiente de interação social que pode ser transposto para momentos em que não seja possível a presença do suporte em questão ${ }^{26}$; solução midiática como um ambiente virtual de coleta de dados para avaliação e decisão clínica direta ${ }^{27}$.

\section{Análise e discussão}

Nesta seção, os resultados obtidos no MS serão discutidos e relacionados ao marco teórico desta pesquisa.

\section{Análise geral}

Os artigos avaliados neste mapeamento sistemático apresentam propostas interdisciplinares de intervenção, com o objetivo de compreender e propor soluções baseadas em evidências para os portadores de transtorno do espectro autista (TEA). O cenário interdisciplinar desta pesquisa pode ser percebido pela variedade de periódicos que foram identificados. Dentre os periódicos, destacam-se o Journal of Developmental and Physical Disabilities com três publicações e o Journal of Autism and Developmental Disorders com cinco publicações.

Apesar da predominância de revistas temáticas de psicologia, áreas como computação, ciência da informação, engenharia e educação física foram contempladas no mapeamento, o que proporcionou uma variedade de perspectivas sobre questões e soluções de pesquisas. Com relação à divisão temporal, nove estudos foram encontrados de 2015, quatro em 2016 e seis foram avaliados em 2017.

Os trabalhos descrevem experimentos em culturas e localidades distintas, como China, Alemanha, Estados Unidos, Austrália e Índia, porém não há relatos nos estudos que assegurem diferenças significativas de acordo com cada um desses países. A verificação do impacto dessas experiências fica em aberto nos artigos analisados, o que fomenta a realização de estudos futuros com esta finalidade.

Outro tópico presente de modo majoritário nos artigos é a dificuldade da transferência das habilidades ensinadas pela solução midiática para o contexto social do indivíduo portador de TEA, bem como a capacidade de generalizar o comportamento que lhe foi representado. A recorrente afirmação sobre a dificuldade de realizar e acompanhar testes por longos períodos, assim como a complexidade de variáveis que envolvem os portadores desse espectro, reflete em pesquisas que alcançaram de modo parcial os resultados pretendidos e apontam a necessidade de prosseguir estudos e experimentações sobre o tema. 


\section{Relação entre as categorias}

A classificação proposta por este mapeamento entre problemas de pesquisa e soluções permite uma visão sobre como as diferentes áreas temáticas e pesquisadores têm direcionado seus estudos. Ao analisar o Gráfico 1, percebe-se a predominância da categoria de problemas nos itens de mentalização ( $\left.\mathrm{P}_{3}\right)$ e habilidades recreativas ( $\left(\mathrm{P}_{4}\right)$. A soma dos estudos desses tópicos resulta em onze artigos e correspondem a $58 \%$ das pesquisas. Em relação às soluções, os estudos em maior evidência concentram-se na categoria de conteúdo por meio dos modelos em vídeo (S1) e produções contextualizadas para o usuário (S2); correspondem a doze estudos que equivalem a $63 \%$ de toda a amostra.

Na intersecção entre as categorias, a modelagem vídeo (S1) aparece em oito pesquisas como a estratégia de intervenção para as questões de métodos $(\mathrm{P} 1)$, habilidades sociais gerais $(\mathrm{P} 2)$, mentalização $\left(\mathrm{P}_{3}\right)$ e recreativas (P4). Além da relação quantitativa, a VM é retratada qualitativamente pelos estudos ${ }^{18,19,23,25,28-31}$ como uma estratégia baseada em evidência de transferência de habilidades e manutenção de comportamentos. Nos estudos avaliados, a expressão Video Modelling foi utilizada para definir cenas de vídeo documentais modeladas por seres humanos. Todavia, é possível reconhecer similitudes entre a VM e as soluções de jogos ou sistemas computacionais que utilizam ambientes e assistentes virtuais como mecanismo para expor modelos de comportamentos e ações. Sobre essa questão, percebe-se que o conceito adotado para a VM pode ser expandido a outros formatos estéticos e tecnológicos, visto que a modelagem em vídeo também ocorre nos jogos ativos ou exergames e que estes contribuem também para o processo de interação com a mídia, já que a ação de recriar o movimento aprendido é registrada por sensores de movimento e mantém relação direta com o conteúdo.

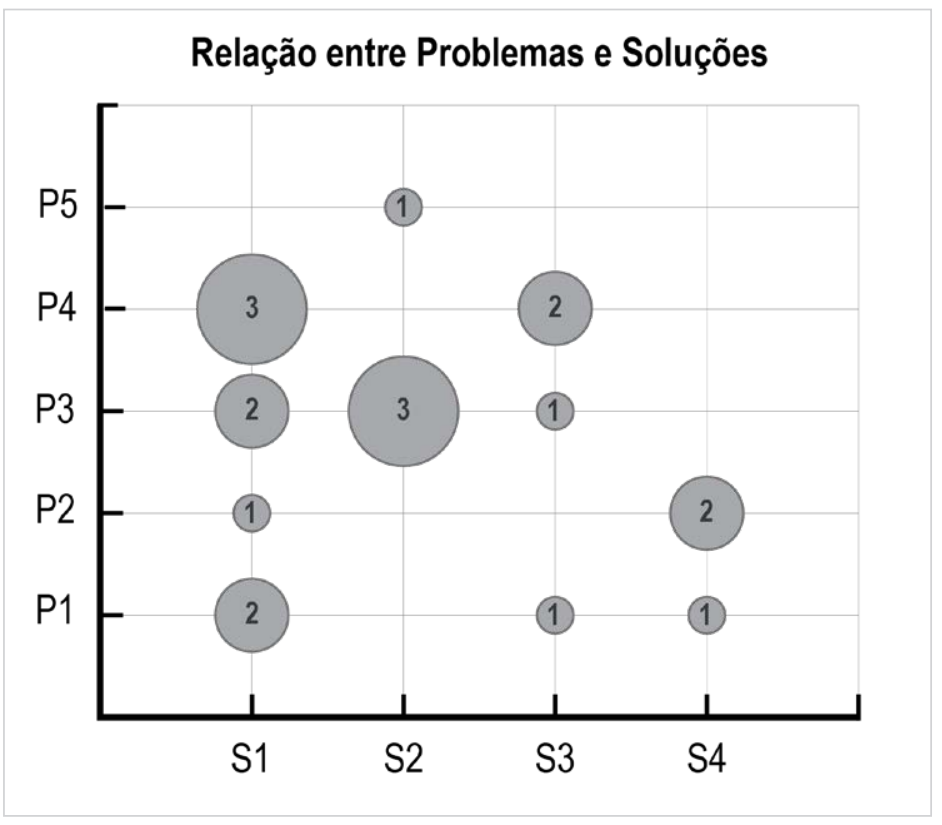

Gráfico 1 - Comparativo entre as categorias

Fonte: Os autores (2019).

Ainda que afinidades conceituais possam ser estabelecidas entre VM, jogos ativos e ambientes virtuais, os relatos encontrados nas pesquisas deste MS sobre a popularidade de adesão e eficácia da VM não mencionam dispositivos e interações complexas. Pelo contrário, a VM é destacada pela sua representação próxima da realidade e pela baixa necessidade de competências e habilidades para interação com a mídia tanto do portador quanto pelo mediador da atividade ${ }^{18}$. Desse modo, abre-se um campo de investigação sobre como a relação entre as affordances, isto é, as condições propiciadas pelo ambiente midiático e as aptidões dos indivíduos portadores impactam na intervenção de ensino e transferência de habilidades modeladas. 


\section{Uso de modelos de atividade}

Como descrito no protocolo Pico deste mapeamento, um dos objetivos desta pesquisa é a verificação do uso de modelos de atividade como estratégia de ensino de habilidades. Entre os estudos avaliados foi identificado o uso majoritário desse paradigma, bem como uma convergência entre as teorias propostas nos estudos ${ }^{5,7}$. Para esses autores, o processo de aprendizagem está relacionado ao contato com mediadores e atividades externas que promovem a relação de modelo e objetivo para que um indivíduo reconheça e internalize o conhecimento ensinado.

Dentro do MS foi identificado o uso de modelos com o objetivo de estimular a imitação de forma integral nas soluções de modelagem em vídeo (S1), uma vez que a proposta central desta intervenção consiste na reprodução de comportamentos para os portadores, com o intuito de estimular a repetição das atividades após a reprodução do conteúdo ${ }^{18,19,23,25,28-31}$. Dialogando com essa abordagem, o jogo ativo proposto no estudo $^{15}$ também apresenta modelos a serem seguidos. Ao acrescentar sensores de movimento, a solução consegue também integrar o processo de imitação no instante de interação com a mídia.

Os estudos ${ }^{20,22}$ apresentam uma solução de interação que pode ser considerada um modelo de interação às ações cognitivas. Essas soluções surgem a partir dos déficits de mentalização e aptidões recreativas dos portadores de TEA e desenvolvem interfaces de interação entre ser humano e computador com o objetivo de estimular tais aptidões. Por exemplo, o estudo ${ }^{22}$ utiliza uma solução de realidade aumentada para conduzir aos portadores de TEA habilidades como substituição de objeto, atribuição de propriedades ausentes e presença de objetos imaginários, já que naturalmente essas variáveis neles são comprometidas. De modo semelhante, o estudo ${ }^{20}$ propõe a utilização de mídias tangíveis para ensinar o processo de emparelhamento de objetos e coordenação mão-olho.

Essa atribuição de modelos externos que impactam o processamento interno pode ser relacionada à teoria da atividade, proposta por Leontyev ${ }^{7}$ Em suas pesquisas, o autor aponta que o processo de internalização da aprendizagem ocorre por meio de uma atividade orientada a um objetivo que, por sua vez, impacta os objetos da atividade, e o desenvolvimento de funções psíquicas e físicas do indivíduo. Somada a essa discussão, a relação proposta pelo estudo ${ }^{5}$ sobre estágios de internalização e complexos de associação, coleção e cadeia nos permite compreender que as soluções que utilizam modelos de atividade desencadeiam, além das modelagens físicas, também as cognitivas.

Por fim, analisando os estudos ${ }^{32,33,35}$, percebe-se que, ao invés de apresentar um modelo para o estímulo, estas pesquisas se encontram no processo de construção de habilidades para desenvolver a capacidade observacional dos portadores, isto é, estimular nos indivíduos a relação de variabilidade e perspectiva de comportamentos e estados emocionais.

\section{Cenários de interação}

Ao analisar os artigos aqui mencionados, percebe-se o uso recorrente do método de design por sondas múltiplas a partir de quatro cenários, ou contextos gerais de interação: C1- Sonda de base; C2- Seção de intervenção; $\mathrm{C}_{3}$ - Manutenção; $\mathrm{C}_{4}$ - Generalização. A sonda de base (C1) é apresentada nos estudos aqui analisados como uma etapa de observação das potencialidades, necessidades e interesses dos portadores. Nesta etapa, as crianças foram avaliadas em escalas clínicas de aptidões motoras, sociais e comportamentais através de atividades estruturadas com e sem suporte midiático. Os estudos utilizam as informações coletadas com dois objetivos: a certificação de equivalência nos indivíduos para o teste e acompanhamento dos resultados após a intervenção. Foi recorrente nas pesquisas a realização desse estágio fora do contexto da solução midiática, proposta com o objetivo de avaliar o cenário social real de aplicação das habilidades. Todavia, estudos que dispunham de uma estrutura tecnológica complexa ${ }^{15,20,22,34}$ 
realizaram testes preliminares com os portadores para identificar se estes dispunham de competências e habilidades para o uso das soluções.

A seção de intervenção (C2) é o instante em que a solução (conteúdo e tecnologia) é apresentada aos indivíduos e eles são convidados a interagir com a mídia. Nessa ocasião os modelos de execução de atividades são apresentados e, por meio de abordagens lúdicas e mediação dos clínicos, ou responsáveis, as habilidades propostas vão sendo trabalhadas. Após o primeiro contato de intervenção, os usuários passam por atividades de aplicação e revisão da modelagem inicial, etapa conhecida como o contexto de manutenção $\left(\mathrm{C}_{3}\right)$. A frequência de seções de manutenção varia de acordo com as aptidões dos portadores, a complexidade da tarefa e, como sugerido por alguns estudos, o suporte midiático adotado ${ }^{18,19,22}$.

Ao atingir índices satisfatórios pré-estabelecidos pela equipe, as crianças dão início a etapa de generalização (C4). Nesse momento, o conjunto de aptidões trabalhadas pela mídia já é reproduzido pela criança, e atividades de aplicação e variação de contexto são introduzidas. No decorrer da intervenção geral da habilidade, os pesquisadores alternam entre os cenários de interação, já que as aptidões dos portadores variam. Nesse sentido, é possível estabelecer uma associação dos cenários com a zona proximal ${ }^{5}$, uma vez que a relação entre as capacidades reais e potenciais de um indivíduo são dinâmicas justamente pela exposição às atividades e à mediação.

Os estudos avaliados neste MS utilizam, de modo geral, esses quatro cenários como etapas metodológicas de aplicação de suas pesquisas. A partir da análise dos artigos, percebe-se que a produção dos sistemas, ao considerar esses cenários previamente, tem a possibilidade de construir um design de interação que aprenda com este percurso e proporcione um ambiente personalizado. Ou seja, se porta como um método de planejamento e criação tanto das affordances dos sistemas para que o usuário atinja seus objetivos, quanto das competências e habilidades que o portador terá como requisito para utilizar a solução.

Além disso, essa construção com foco nos diferentes estágios de interação permite a compreensão de que o indivíduo portador de TEA assuma ao longo dos processos diferentes papéis em relação à mídia de intervenção. O que impacta novamente a projeção de um design audiovisual que considere os cenários, os indivíduos, as affordances e principalmente, os processos de interação e progressão do aprendizado.

\section{Métricas de avaliação clínica}

$\mathrm{Na}$ avaliação dos métodos, materiais e resultados dos artigos, foi identificado o uso recorrente de múltiplas medidas clínicas tanto para aferir o diagnóstico nos participantes quanto para estabelecer métricas de acompanhamento durante o processo de intervenção. Essas métricas são utilizadas pelos pesquisadores ao longo de todos os cenários de interação e atuam como suporte à tomada de decisão clínica, bem como na mineração de dados para o suporte e criação dos sistemas. Ao todo, foram encontradas trinta e cinco abordagens para a medição de competências e habilidades que estão dispostas de modo variado entre os estudos, pois algumas pesquisas utilizam apenas uma escala, enquanto outras intercalam dez. As métricas adotadas variam de estratégias para avaliação qualitativa, até processos quantitativos de medição de atenção, interação social, nível de reconhecimento de expressões, imagens, sons, capacidades motoras, entre outros.

Entre os parâmetros recorrentes para o diagnóstico dos indivíduos como portadores de TEA estão o Diagnostic and Statistical Manual of Mental Disorders (DSM- IV/V) aplicado a oito pesquisas ${ }^{15,19,21,25,29-32}$, Autism Diagnostic Observation Schedule (ADOS-2/G) aplicado a cinco pesquisas ${ }^{15,21,30,32,33}$ e Autism Diagnostic Interview- Revised (ADI-R) adotado por três pesquisas ${ }^{33,21,30}$, (Gráfico 2). Os estudos ${ }^{20,24,26}$ não apresentaram o uso de protocolos clínicos formais de medição. Nessas pesquisas, a validação do diagnóstico dos participantes foi reaproveitada de aferições médicas anteriores. Todavia, os estudos descreveram por meio dos cenários de investigação (sonda de base) as habilidades reais dos indivíduos e a progressão de aprendizado das aptidões esperadas. 


\section{Recorrência dentre os estudos}

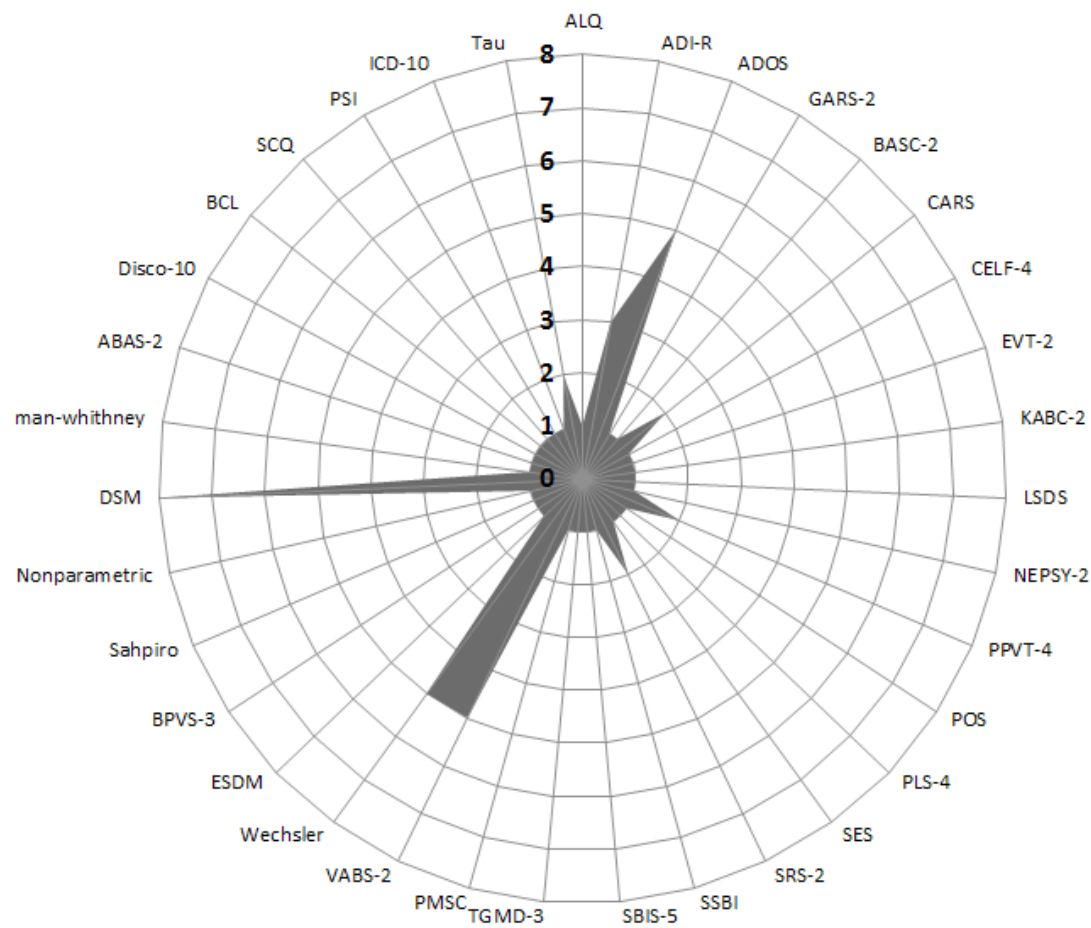

Gráfico 2 - Métricas clínicas para medir competências e habilidades Fonte: Os autores (2019).

A natureza interdisciplinar encontrada neste mapeamento é novamente representada pela variedade das escalas identificadas. Devido a esse cenário, foram encontradas situações em que ora um protocolo é utilizado para diagnóstico, ora para acompanhamento. A respeito das escalas para acompanhamento, as medidas recorrentes são: Vineland Adaptive Behavior Scales (VABS-II) e Wechsler Intelligence Scales III - IV, (Gráfico 2). Na maior parte dos estudos, o uso das escalas foi mediado por profissionais que acompanharam simultaneamente as seções, presencialmente ou por análise de registros em vídeo das atividades. Apenas o estudo ${ }^{33}$ apresentou a proposta de incorporar o acompanhamento das escalas integradas ao uso da aplicação; todavia, as soluções que dispunham de sistemas integrados a sensores de voz, movimento e rastreamento de olhar utilizaram os registros de interação como métrica de avaliação complementar 15,22,34.

A utilização de medidas clínicas, como estratégia para quantificar e acompanhar a evolução do aprendizado de modo integrado às funcionalidades do sistema audiovisual, é vista por esse MS como uma estratégia potencial, pois à medida que integra o processo de coleta, análise e processamento de dados, dispõe da possibilidade de inserir filtros de personalização e recomendação de conteúdo para atingir os requisitos unitários de cada portador. Sendo assim, se faz necessário investigar como as escalas podem servir para averiguar as habilidades necessárias ao uso das soluções, bem como quais aptidões são melhores trabalhadas por formatos, sistemas e interfaces de interação.

\section{Conclusão}

Este trabalho apresentou um mapeamento sistemático da utilização de sistemas audiovisuais no processo de ensino de habilidades para portadores do transtorno do espectro autista. Com o objetivo de atender às questões de pesquisa, foi conduzido um mapeamento que revisou artigos de quatro bases de dados, no período de 2015 a 2017, classificando as informações de acordo com a perspectiva de problemas e soluções de pesquisa. 
De modo geral, os estudos concentram-se em problemas sobre métodos de intervenção, habilidades sociais gerais, habilidades de mentalização, recreativas e acadêmicas. Já as soluções propõem estudos com foco em conteúdo, tecnologia e contexto. Após a análise dos dados, percebe-se que os sistemas audiovisuais adotados variam entre vídeos, jogos e softwares, e são utilizados em quatro grandes cenários de interação (sonda de base, intervenção, manutenção e generalização) com o objetivo de compreender as capacidades reais e potenciais dos usuários e apresentar um conteúdo que modele emoções e comportamentos. Na intersecção das categorias, a solução modelagem em vídeo (S1) apresenta maior frequência e evidência de resultado, além de se relacionar com quatro dos cinco problemas mapeados. Em relação ao processo de medição e acompanhamento do aprendizado, foi identificado o uso de trinta e cinco escalas de medidas clínicas que foram utilizadas para o diagnóstico e acompanhamento das intervenções. Apesar dos resultados em linhas gerais terem sido parciais, a evidência de eficácia das estratégias de intervenções é destacada pelos autores.

Por fim, percebe-se que as intervenções midiatizadas, que variam desde o consumo passivo como audiência (vídeo), passando pelo uso de interfaces em que há interação entre homem e máquina até a interação e manipulação do conteúdo por meio de jogos ativos, podem expandir sua eficácia de intervenção ao incorporarem as lacunas e sugestões de pesquisas apontadas pelos autores e integrarem os cenários de interação, papéis dos portadores, mineração dos dados clínicos, fins terapêuticos, affordances de cada mídia e as competências e habilidades dos portadores. Tudo isso com o objetivo de estabelecer um design de produtos audiovisuais para o treinamento de habilidades sociais.

\section{Referências}

1. Toscano R, Becker V. Sistemas audiovisuais inteligentes: um levantamento das práticas que apontam a dinâmica da narrativa responsiva e generativa [Internet]. In: $18^{\circ}$ Congresso de Ciências da Comunicação na Região Nordeste; 2016 jul 07-09; Caruaru. Pernambuco: Intercom; 2016 [citado em 2019 maio 10]. Disponível em: http://bit.do/eR4zr

2. Mintz M. Evolution in the understanding of autism spectrum disorder: historical perspective. Indian J Pediatr. 2017 Jan 7;84(1):44-52. doi: https://doi.org/10.1007/s12098-016-2080-8

3. Passerino LM, Santarosa LC. Uso de ferramentas síncronas para análise da interação social em sujeitos com autismo: Um estudo de caso. RENOTE. 2005;3(1):1-11. doi: https://doi.org/10.22456/1679-1916.13728

4. American Psychiatric Association. Manual diagnóstico e estatístico de transtornos mentais: DSM-5. Porto Alegre: Artmed; 2014.

5. Vygotsky LS. Pensamento e linguagem. São Paulo: Martins Fontes; 1989.

6. Fonseca V. Cognição, neuropsicologia e aprendizagem. 7 ed. Petrópolis: Editora Vozes; 2015.

7. Leontyev AN. Activity and consciousness [Internet]. In: Philosophy in the USSR, Problems of Dialectical Materialism. Moscow: Progress Publishers; 1977 [cited 2019 May 10]. 180-201. Available from: http:// bit.do/eR4Cb

8. Sirlene M, Schlickmann P. Teoria da atividade e teoria da relevância: um estudo introdutório sobre suas implicações no processo ensino-aprendizagem. Ling (Dis)curso. 2002;3(1):211-24.

9. Greiner C, Katz H. Corpo e processos de comunicação. Front Estud Midiáticos. 2001;3(1):63-74.

10. Pellanda NMC, Demoly KRA. As tecnologias touch: corpo, cognição e subjetividade. Psicol Clin [Internet]. 2014 jun. [citado em 2019 maio 10];26(1):69-89. Disponível em: http://bit.do/eR4CL. doi: http://dx.doi. org/10.1590/S0103-56652014000100006

11. Timo ALR, Maia NVR, Ribeiro PC. Déficit de imitação e autismo: uma revisão. Psicol USP [Internet]. 2011[citado em 2019 maio 10];22(4). Disponível em: http://bit.do/eR4DY. doi: http://dx.doi. org/10.1590/S0103-65642011005000035

12. Kitchenham B, Charters S. Guidelines for performing Systematic Literature reviews in software Engineering Version 2.3. Engineering [Internet]. 2007 [cited 2019 May 10];45(4ve):1051. Available from: http://bit.do/eR4Hj 
13. Souza DM, Batista MHS, Barbosa EF. Problemas e dificuldades no ensino e na aprendizagem de programação: um mapeamento sistemático. Rev Bras Informática Educ. 2016;24:39-52. doi: http:// dx.doi.org/10.5753/rbie.2016.24.1.39

14. Anderson-Hanley C, Tureck, Schneiderman. Autism and exergaming: effects on repetitive behaviors and cognition. Psychol Res Behav Manag [Internet]. 2011 Sept [cited 2019 May 10];4:129-37. Available from: http://bit.do/eR4Jt doi: http://dx.doi.org/10.2147/PRBM.S24016

15. Edwards J, Jeffrey S, May T, Rinehart NJ, Barnett M. Does plaiyng a sports active video game improve object control skills of children with autism spectrum disorder? J Sport Heal Sci [Internet]. 2016 [cited 2019 May 10];4(1):17-24. Available from: http://bit.do/eR4K9 doi: https://doi.org/10.1016/j. jshs.2016.09.004

16. Ferguson MB, Anderson-Hanley PC, Mazurek MO, Parsons S, Warren Z. Game interventions for autism spectrum disorder. Games Health J [Internet]. 2012 [cited 2019 May 10];1(4):248-53. Available from: http://bit.do/eR4LX doi: https://doi.org/10.1089/g4h.2012.0717

17. Collins DM, Medical T, Mowafaghian D, Health B, Columbia B, Hilton CL, et al. Exergaming to improve physical and mental fitness in children and adolescents with autism spectrum disorders: pilot study clinmed. Int J Sport Exerc Med [Internet]. 2015 [cited 2019 May 10];1(3):1-6. Available from: http:// bit.do/eR4NX

18. Miltenberger CA, Charlop MH. The comparative effectiveness of portable video modeling vs. traditional video modeling interventions with children with autism spectrum disorders. J Dev Phys Disabil. $2015 ; 27(3): 341-58$

19. Malmberg DB, Charlop MH, Gershfeld SJ. A Two experiment treatment comparison study: teaching social skills to children with autism spectrum disorder. J Dev Phys Disabil. 2015;27(3):375-92.

20. Durango I, Carrascosa A, Gallud JA, Penichet VMR. Interactive fruit panel (IFP): a tangible serious game for children with special needs to learn an alternative communication system. Univers Access Inf Soc [Internet]. 2018 [cited 2019 May 10].17(1):51-65. Available from: http://bit.do/eR4Qm

21. Malinverni L, Mora-Guiard J, Padillo V, Valero L, Hervás A, Pares N. An inclusive design approach for developing video games for children with autism spectrum disorder. Comput Human Behav. 2017;71:535-49. doi: https://doi.org/10.1016/j.chb.2016.01.018

22. Zhen B, Blackwell AF, Coulouris G. Using augmented reality to elicit pretend play for children with autism. IEEE Trans Vis Comput Graph [Internet]. 2015 [cited 2019 May 10];21(5): 598-610. Available from: http://bit.do/eR4S9

23. Spriggs AD, Gast DL, Knight VF. Video modeling and observational learning to teach gaming access to students with ASD. J Autism Dev Disord. 2016;46(9):2845-58. doi: https://doi.org/10.1007/ s10803-016-2824-3

24. Eder MS, L. Diaz JM, S. Madela JR, U. Mag-usara M, M. Sabellano DD. Fill me app: an interactive mobile game application for children with autism. Int J Interact Mob Technol [Internet]. 2016 [cited 2019 May 10];10(3):59. Available from: http://online-journals.org/index.php/i-jim/article/view/5553

25. Macpherson K, Charlop MH, Miltenberger CA. Using portable video modeling technology to increase the compliment behaviors of children with autism during athletic group play. J Autism Dev Disord. 2015;45(12):3836-45. doi: https://doi.org/10.1007/s10803-014-2072-3

26. Özen A. Effectiveness of siblings-delivered ipad game activities in teaching social interaction skills to children with autism spectrum disorders. Kuram ve Uygulamada Egit Bilim. 2015;15(5):1287-303.

27. Bartolome NA, Zapirain BG. Cognitive rehabilitation system for children with autism spectrum disorder using serious games: a pilot study. Biomed Mater Eng. 2015;26:S811-24.

28. Jung S, Sainato DM. Teaching games to young children with autism spectrum disorder using special interests and video modelling. J Intellect Dev Disabil [Internet]. 2015 [cited 2019 May 10] [cited 2019 May 10];40(2):198-212. Available from: http://bit.do/eR4Vt

29. Kourassanis J, Jones EA, Fienup DM. Peer-video modeling: teaching chained social game behaviors to children with ASD. J Dev Phys Disabil. 2014;27(1):25-36. doi: https://doi.org/10.1007/s10882-014-9399-8

30. Ryan C, Furley P, Mulhall K. Judgments of nonverbal behaviour by children with high-functioning autism spectrum disorder: can they detect signs of winning and losing from brief video clips? J Autism Dev Disord. 2016;46(9):2916-23. doi: https://doi.org/10.1007/s10803-016-2839-9 
31. Lee SY, Lo Y, Lo Y. Teaching functional play skills to a young child with autism spectrum disorder through video self-modeling. J Autism Dev Disord [Internet]. 2017 [cited 2019 May 10]; 47(8):2295-2306. Available from: http://bit.do/eR5UV

32. Fridenson-Hayo S, Berggren S, Lassalle A, Tal S, Pigat D, Meir-Goren N, et al. "Emotiplay": a serious game for learning about emotions in children with autism: results of a cross-cultural evaluation. Eur Child Adolesc Psychiatry. 2017;26(8)1-14. doi: https://doi.org/10.1007/s00787-017-0968-0

33. Jouen A-L, Narzisi A, Xavier J, Tilmont E, Bodeau N, Bono V, et al. GOLIAH (Gaming Open Library for Intervention in Autism at Home): a 6-month single blind matched controlled exploratory study. Child Adolesc Psychiatry Ment Health [Internet]. 2017 [cited 2019 May 10]; 11:17. Available from: http://bit. do/eR5VJ doi: https://doi.org/10.1186/s13034-017-0154-7

34. Rice LM, Wall CA, Fogel A, Shic F. Computer-assisted face processing instruction improves emotion recognition, mentalizing, and social skills in students with ASD. J Autism Dev Disord [Internet]. 2015 [cited 2019 May 10];45(7): 2176-86. Available from: http://bit.do/eR5Wg_doi: https://doi.org/10.1007/ s10803-015-2380-2

35. Craig AB, Brown ER, Upright J, DeRosier ME. Enhancing children's social emotional functioning through virtual game-based delivery of social skills training. J Child Fam Stud. 2016; 25(3):959-68. 